\title{
Gate-controlled spin extraction from topological insulator surfaces
}

\author{
Ali Asgharpour $\odot,{ }^{1}$ Cosimo Gorini $\odot,{ }^{2}$ Sven Essert, ${ }^{2}$ Klaus Richter, ${ }^{2}$ and İnanç Adagideli ${ }^{1, *}$ \\ ${ }^{1}$ Faculty of Engineering and Natural Sciences, Sabancı University, Orhanlı Tuzla 34956, Turkey \\ ${ }^{2}$ Institut für Theoretische Physik, Universität Regensburg, 93040 Regensburg, Germany
}

(Received 6 February 2020; accepted 9 June 2020; published 1 July 2020)

\begin{abstract}
Spin-momentum locking, a key property of the surface states of three-dimensional topological insulators (3DTIs), provides a new avenue for spintronics applications. One consequence of spin-momentum locking is the induction of surface spin accumulations due to applied electric fields. In this paper, we investigate the extraction of such electrically induced spins from their host TI material into adjoining conventional, hence topologically trivial, materials that are commonly used in electronics devices. We focus on effective Hamiltonians for bismuth-based 3DTI materials in the $\mathrm{Bi}_{2} \mathrm{Se}_{3}$ family, and numerically explore the geometries for extracting current-induced spins from a TI surface. In particular, we consider a device geometry in which a side pocket is attached to various faces of a 3DTI quantum wire and show that it is possible to create current-induced spin accumulations in these topologically trivial side pockets. We further study how such spin extraction depends on geometry and material parameters, and find that electron-hole degrees of freedom can be utilized to control the polarization of the extracted spins by an applied gate voltage.
\end{abstract}

DOI: 10.1103/PhysRevB.102.035401

\section{INTRODUCTION}

The push towards the utilization of the electron's spin degree of freedom in common electronic devices, which are conventionally based on the manipulation of the electron charge, has matured to the field called spintronics [1]. The various lines of research in this field not only comprise questions of fundamental interest in spin physics but also focus on applications. Possible advantages of utilizing spin-based elements in comparison to charge-based electronic devices might be low power consumption and less heat dissipation, as well as more compact and faster reading or writing of data.

The ferromagnets [2-4] are the mainstream materials used in spintronics where the ferromagnetic exchange interaction causes the spin dependency of transport, allowing the creation, manipulation, and detection of spins. However, after the celebrated Datta-Das spin transistor proposal [5], it became clear that spin-orbit interaction can also be utilized for spin manipulation in electronic devices. As the Datta-Das setting still requires ferromagnetic leads, a parallel approach utilizing materials without intrinsic magnetism, such as paramagnetic metals and semiconductors with only spin-orbit coupling [6-8], has become an attractive alternative.

Various methods of spintronics implementations without ferromagnets have emerged and developed over recent years [9-19]. These methods are commonly based on (i) the spin Hall effect [14], where an applied electric current generates a transverse spin current, and (ii) Edelstein (or inverse spin galvanic) effect $[9,20]$, where an applied electrical current generates a nonzero spin accumulation. Once generated, as these spins drive spintronics circuits, they need to be further manipulated and ultimately detected. For detection, inverse

\footnotetext{
*adagideli@sabanciuniv.edu
}

effects corresponding to those mentioned above, namely, the inverse spin Hall effect [21-25] and spin galvanic effect (SGE) [26-30], have been successfully utilized. Main methods for spin manipulation are based on exchange and Zeeman fields or spin-orbit coupling to induce spin precession. However, weak coupling requires long length scales over which the induced spins need to remain coherent. This is an issue as spin precession lengths are usually comparable to spin relaxation or dephasing lengths. Furthermore, the spin-orbit coupling needs to be controlled over the precession (hence manipulation) region, while spin generation in part of the circuit needs to remain unaffected. Hence, in order to close the creation, manipulation, and detection cycle reliably, additional electrical methods for spin manipulation are desirable.

In this paper, we consider a mechanism in topological insulators (TIs) that allows for local and all-electrical control of electrically generated spins with gates. In most spintronics (or spin-orbitronics) platforms, charge carriers are of a given type, either electron or hole, implying that local application of gates equally couples to both spin species. In others where electron and hole pockets might coexist, there is no coherence between the electron or hole degree of freedom and the spin degree of freedom. As a consequence, electric gates cannot locally control local spin accumulations in conventional spintronics and spin-orbitronics platforms. On the other hand, the surface (or edge) of three-dimensional (two-dimensional) TIs features both electron and hole degrees of freedom as well as spin-orbit coupling. Applied gates control the local potential, which couples oppositely to electrons and holes, and spinorbit coupling allows for spin dependency of electron-hole degrees of freedom. We demonstrate below that this joint property allows for electronic control of spins locally within a region much smaller than the spin precession length, the length scale over which spins can be manipulated in conventional spintronics applications [1]. 


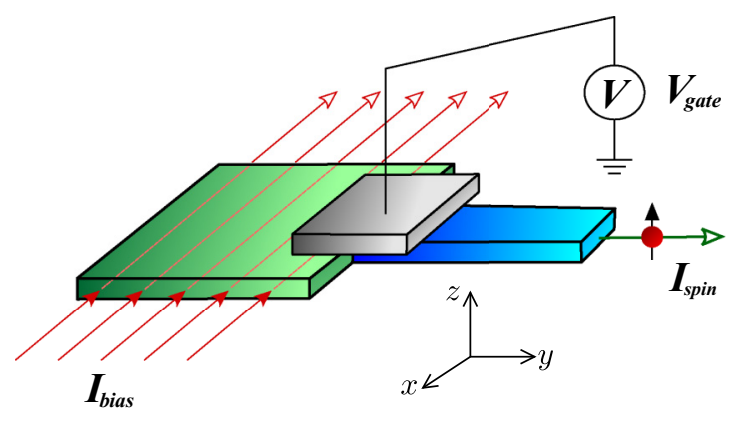

FIG. 1. Slab of a topological insulator (green), current biased with $I_{\text {bias }}$. The induced spin accumulation at the boundaries can be injected into a side contact (blue). A gate potential $V_{\text {gate }}$ can be tuned to control the spin polarization of the spin injected current.

As an explicit example, we consider three-dimensional TI (3DTI) materials of the $\mathrm{Bi}_{2} \mathrm{Se}_{3}$ family the effective model of which is extensively discussed in the literature [31-34]. Qualitatively, our conclusions should apply also to strained (3D) $\mathrm{HgTe}$, though an equally successful effective model for such a system is still missing. We focus on a particular geometry (sketched in Fig. 1) and demonstrate how the spin extraction can be controlled in a region smaller than the spin precession length. In this geometry, the spins are generated by the spin galvanic effect at the surface of the TI. By attaching a side pocket and tuning the chemical potential on the pocket by an applied gate voltage, we demonstrate that the extracted spins can change their polarization, regardless of the generated spins on the TI side.

Our paper is organized as follows. In Sec. II A, we outline the effective surface Hamiltonian of a 3DTI and the corresponding spin operators. We then present the inverse spin galvanic effect (ISGE), also known as the Edelstein effect, through Kubo formalism in Sec. II B. Different names addressing the same phenomenon are used in the literature depending on the context. In Sec. IIC, we state an ISGE paradox with its solution for the surfaces of a 3DTI. Next, we discuss the model and the method proposed for extracting spin from surfaces of a 3DTI in Sec. III A. In Sec. III B, we derive the spin behavior on the 3DTI surfaces, which we show to be in close agreement with our numerical simulations. In Sec. IIIC, we demonstrate how to extract spins from 3DTI surfaces and how to manipulate their polarization through a gate potential. We close with concluding remarks in Sec. IV.

\section{A SPIN-GALVANIC PARADOX AND ITS SOLUTION}

\section{A. Setting the stage}

Consider a finite crystal of an anisotropic 3DTI material, such as $\mathrm{Bi}_{2} \mathrm{Se}_{3}$, which in its TI phase hosts topologically protected metallic surface states. The existence of these states, described by a single Dirac cone, was confirmed experimentally by angle-resolved photoemission spectroscopy $[35,36]$ and scanning tunneling spectroscopy [37-41] measurements. Further experiments confirmed the helical nature of such surface states [42]. The anisotropy of these materials implies that the topological metallic states existing on the different crystal faces will be described by Dirac-like effective Hamiltonians featuring different spin structures [31-33]. We are interested in the consequences of the anisotropy of these materials on the ISGE [9,20,43]; for recent discussions see [44-46].

The states of the two-dimensional (2D) helical surfaces of $\mathrm{Bi}_{2} \mathrm{Se}_{3}$ are admixtures of electron- and holelike states of different parity $( \pm)$ and spin $(\uparrow \downarrow)$, coming from $\mathrm{Bi}$ and $\mathrm{Se}$ $p_{z}$ orbitals, $\left|P 1_{z}^{+}, \uparrow \downarrow\right\rangle$ and $\left|P 2_{z}^{-}, \uparrow \downarrow\right\rangle$, respectively [34]. As a consequence, the real spin content of such states does not necessarily coincide with the pseudospin degrees of freedom used to label them. Hence, $\sigma_{i}(i=x, y, z)$ denote the Pauli operators corresponding to the two bands at the surface (the pseudospin), while $s_{i}$ are the spin operators within this restricted Hilbert space. The effective spin operators for any surface orientation are obtained by projecting the full spin operators of the combined electron and hole bands onto the relevant surface states (see Appendix A). This projection misses key physics which we later utilize to electrically control spin accumulations. The most commonly "known" low-energy effective Hamiltonian for the topological surface state is that of the "top" and "bottom" surfaces in the growth direction, which we choose to be in the $\hat{\mathbf{z}}$ direction:

$$
H^{ \pm \hat{\mathbf{z}}}=E_{0}(\hat{\mathbf{z}})+v_{F}(\hat{\mathbf{z}})(\mathbf{k} \times \hat{\mathbf{z}}) \cdot \boldsymbol{\sigma},
$$

where $E_{0}(\hat{\mathbf{z}})$ is the energy of the Dirac point, $v_{F}(\hat{\mathbf{z}})$ is the corresponding Fermi velocity, and \pm refers to the surface normals pointing away from the bulk. In this case, the spin and the pseudospin operators are the same:

$$
\mathbf{s}=\sigma
$$

This identification as well as the rotational symmetry, however, is lost at the side surfaces:

$$
H^{ \pm \hat{\mathbf{y}}}=E_{0}(\hat{\mathbf{y}}) \pm v_{F, x}(\hat{\mathbf{y}}) k_{x} \sigma_{z} \mp v_{F, z}(\hat{\mathbf{y}}) k_{z} \sigma_{x},
$$

where $E_{0}(\hat{\mathbf{y}})$ is the energy of the Dirac point, and $v_{F, x}(\hat{\mathbf{y}})$ and $v_{F, z}(\hat{\mathbf{y}})$ are the corresponding Fermi velocity in the $x$ and $z$ directions, respectively. In this case, while the $x$ components of the spin and the pseudospin operators are the same, they are merely proportional in the $\hat{\mathbf{y}}$ and $\hat{\mathbf{z}}$ surfaces with the proportionality parameter $\eta$ :

$$
s_{x}=\sigma_{x}, \quad s_{y}=\eta \sigma_{y}, \quad s_{z}=\eta \sigma_{z} .
$$

For completeness, we express the $\pm \hat{\mathbf{x}}$ surface Hamiltonian as

$$
\begin{gathered}
H^{ \pm \hat{\mathbf{x}}}=E_{0}(\hat{\mathbf{x}}) \mp v_{F, y}(\hat{\mathbf{x}}) k_{y} \sigma_{z} \pm v_{F, z}(\hat{\mathbf{x}}) k_{z} \sigma_{y}, \\
s_{x}=\eta \sigma_{x}, \quad s_{y}=\sigma_{y}, \quad s_{z}=\eta \sigma_{z},
\end{gathered}
$$

where $E_{0}(\hat{\mathbf{x}})=E_{0}(\hat{\mathbf{y}}), v_{F, y}(\hat{\mathbf{x}})$, and $v_{F, z}(\hat{\mathbf{x}})$ are the Fermi velocities in the $y$ and $z$ directions, respectively. To summarize, the real spin coincides with the Pauli matrices $\sigma_{i}, i=x, y, z$ of the pseudospin only on the $\pm \hat{\mathbf{z}}$ surface. In particular, if $\eta \rightarrow 0$, the surface states on the $\pm \hat{\mathbf{y}}$ side have $s_{y}=0, s_{z}=0$. This point is crucial, as we discuss below.

\section{B. Spin galvanic basics}

We consider the spin accumulation, $s_{z}(\omega)$, generated in response to an applied electric field $E_{x}$ in a spin-orbit coupled 
2D system lying in the $\hat{\mathbf{x}}-\hat{\mathbf{z}}$ plane-corresponding to the side surfaces $\pm \hat{\mathbf{y}}$. The ISGE can be written in Kubo form [10] as

$$
\begin{gathered}
s_{z}(\omega)=\sigma_{\mathrm{ISGE}}(\omega) E_{x}(\omega) \\
=\left\langle\left\langle s_{z} ; J_{x}\right\rangle\right\rangle A_{x}(\omega),
\end{gathered}
$$

where $\left\langle\left\langle s_{z} ; J_{x}\right\rangle\right\rangle=\frac{-i}{\hbar} \int_{0}^{t}\left\langle\left[s_{z}(t), J_{x}(0)\right]\right\rangle e^{i \omega t} d t$ is the Kubo linear response kernel, $A$ is the vector potential, and $\sigma_{\text {ISGE }}$ is the frequency-dependent ISGE conductivity. Thus

$$
\sigma_{\mathrm{ISGE}}(\omega)=\frac{\left\langle\left\langle s_{z} ; J_{x}\right\rangle\right\rangle}{i \omega} .
$$

Its Onsager reciprocal effect, the SGE, reads [30]

$$
\begin{gathered}
J_{x}(\omega)=\sigma_{\mathrm{SGE}}(\omega) \dot{B}_{z}(\omega) \\
=\left\langle\left\langle J_{x} ; s_{z}\right\rangle\right\rangle B_{z}(\omega),
\end{gathered}
$$

yielding

$$
\sigma_{\mathrm{SGE}}(\omega)=\frac{\left\langle\left\langle J_{x} ; s_{z}\right\rangle\right\rangle}{i \omega} .
$$

In Eq. (11) $\dot{B}$ is the time derivative of the magnetic field which generates the nonequilibrium $s_{z}$ leading to the SGE.

\section{Spin galvanic effect on the surface of a 3DTI}

As we stressed above, the relation between the pseudospin $\sigma$ and the real spin $\mathbf{s}$ on the 3DTI surface can be anisotropic. The two quantities are identical on the $\pm \hat{\mathbf{z}}$ surfaces, and hence there is no ambiguity in calculating the ISGE and the SGE on the surfaces. However, on the $\hat{\mathbf{y}}$ surfaces

$$
s_{z}=\eta \sigma_{z} .
$$

On the surface of the TI, spin and charge or momentum are locked. To be explicit we assume

$$
J_{x}=v_{F, x}(\hat{\mathbf{y}}) \sigma_{z}
$$

with $v_{F, x}(\hat{\mathbf{y}})$ the Fermi velocity in the $x$ direction [see Eqs. (1)(3)]. From Eqs. (13) and (14) one gets

$$
J_{x}=\frac{v_{F, x}(\hat{\mathbf{y}})}{\eta} s_{z} .
$$

Equation (15) seems to imply a divergent ("colossal") SGE for $\eta \rightarrow 0$, while the ISGE should vanish.

This apparent paradox is resolved by judiciously inspecting the SGE and ISGE linear response kernels. First, for the SGE one has

$$
\begin{gathered}
J_{x}=\frac{\left\langle\left\langle J_{x} ; s_{z}\right\rangle\right\rangle}{i \omega} B_{z}(\omega) \\
=\eta v_{F, x}(\hat{\mathbf{y}}) \underbrace{\frac{\left\langle\left\langle\sigma_{z} ; \sigma_{z}\right\rangle\right\rangle}{i \omega}}_{L_{\sigma \sigma}} B_{z}(\omega),
\end{gathered}
$$

which tends to zero for $\eta \rightarrow 0$ as it should: The pseudospinpseudospin response function $L_{\sigma \sigma}$ defined above has no divergencies. Similarly for the ISGE holds

$$
s_{z}=\frac{\left\langle\left\langle s_{z} ; J_{x}\right\rangle\right\rangle}{i \omega} E_{x}(\omega)
$$

$$
=\eta v_{F, x}(\hat{\mathbf{y}}) \underbrace{\frac{\left\langle\left\langle\sigma_{z} ; \sigma_{z}\right\rangle\right\rangle}{i \omega}}_{L_{\sigma \sigma}} E_{x}(\omega)
$$

which is given by the same response function $L_{\sigma \sigma}$ and again vanishes in the $\eta \rightarrow 0$ limit.

\section{SPIN EXTRACTION FROM 3DTI SURFACES}

Even though it turns out that there is no paradox in the form of a divergent SGE response, there are interesting consequences when considering $\eta \rightarrow 0$. In particular, as we show below, it is possible to extract current-induced spins from the side surfaces even if these are not spin polarized. The main idea is the following: at the side surfaces of a TI, an analytical examination of the nonequilibrium population of the $k_{x}$ states (induced by, say, an applied bias) reveals their composition to be a mixture of spin-up electronlike and spin-down holelike quasiparticles the spins of which partially cancel each other. This is the origin of the parameter $\eta \neq 1$ in general. In the limit $D_{2} \rightarrow 0$ (hence $\eta \rightarrow 0$ ) the cancellation is perfect. Therefore, it suffices to contact the surface with a "pocket" containing electrons or holes-in practice, a gated semiconductor-so that only the spin-polarized electron- or holelike part of the surface state will leak out of the TI. A side pocket or lead thus acts as a gate-tunable spin extractor: The sign of the extracted spins can be reversed by simply switching the pocket polarity from $n$ to $p$ type or vice versa, allowing for local electrical control of spin polarization. Note the crucial observation that the size of the region where the spin is reversed can be shorter than the spin precession length (see Fig. 7 below).

\section{A. Model and method}

In the rest of this section, we further study the spin extraction effect through analytical and numerical means for 3DTI nanowires. The wires are described by a 3D effective Hamiltonian which captures the basic low-energy properties of the $\mathrm{Bi}_{2} \mathrm{Se}_{3}$ family, including, e.g., $\mathrm{Bi}_{2} \mathrm{Se}_{3}, \mathrm{Bi}_{2} \mathrm{Te}_{3}$, and $\mathrm{Sb}_{2} \mathrm{Te}_{3}$ materials $[34,47]$ :

$$
\begin{aligned}
H^{3 D}= & E(\mathbf{k})\left(\sigma_{0} \tau_{0}\right)+M(\mathbf{k})\left(\sigma_{0} \tau_{z}\right)+A_{1} \sin k_{z}\left(\sigma_{z} \tau_{x}\right) \\
& +A_{2}\left[\sin k_{x}\left(\sigma_{x} \tau_{x}\right)+\sin k_{y}\left(\sigma_{y} \tau_{x}\right)\right],
\end{aligned}
$$

where

$$
\begin{aligned}
M(\mathbf{k}) & =M_{0}-2 B_{2}\left(2-\cos k_{x}-\cos k_{y}\right)-2 B_{1}\left(1-\cos k_{z}\right), \\
E(\mathbf{k}) & =C+2 D_{2}\left(2-\cos k_{x}-\cos k_{y}\right)+2 D_{1}\left(1-\cos k_{z}\right) .
\end{aligned}
$$

Here, $\sigma_{x, y, z}$ and $\tau_{x, y, z}$ are the Pauli matrices, and $\sigma_{0}$ and $\tau_{0}$ are the $2 \times 2$ identity matrices in spin and orbital space, respectively. If $\left(M_{0} / B_{1}>0\right)$ then the system is in the topologically nontrivial phase and Dirac-like surface states form within the bulk band gap. For a wire, due to the size quantization around the wire, the surface states form one-dimensional (1D) channels and the lowest 1D subband is gapped due to its nontrivial Berry phase $[48,49]$.

In order to find the current-induced spin polarization on the 3DTI nanowire surfaces, we need the spin operators expressed in the basis used to represent Eq. (20). The basis states are hybridized states of the $\mathrm{Se}$ and $\mathrm{Bi} p$ orbitals with even $(+)$ 
and odd (-) parities and spins up $(\uparrow)$ and down $(\downarrow)$, namely, $\left|P 1_{z}^{+}, \uparrow\right\rangle,-i\left|P 2_{z}^{-}, \uparrow\right\rangle,\left|P 1_{z}^{+}, \downarrow\right\rangle$, and $i\left|P 2_{z}^{-}, \downarrow\right\rangle$, in that order. Then the spin operators in the basis of bulk states are given by [33]

$$
S_{x}=\sigma_{x} \tau_{z}, \quad S_{y}=\sigma_{y} \tau_{z}, \quad S_{z}=\sigma_{z} \tau_{0} .
$$

Using the explicit forms of the spin operators, Eqs. (21), we generalize the Kubo response kernel of the effective 2D surface model of the previous section to the more realistic $3 \mathrm{D}$ model (20):

$$
\begin{gathered}
S_{z}(\omega)=\sigma_{\mathrm{ISGE}}(\omega) E_{y}(\omega) \\
=\left\langle\left\langle S_{z} ; J_{y}\right\rangle\right\rangle A_{y}(\omega)
\end{gathered}
$$

with $S_{z}=\sigma_{z} \tau_{0}$.

The effective surface description is obtained by projecting into the space spanned by the surface modes. One thus obtains the effective surface spin and Hamiltonian operators (see Appendix A). These surface Hamiltonians and modes for electrons on 3DTI faces defined by their normals $\pm \hat{\mathbf{x}}, \pm \hat{\mathbf{y}}, \pm \hat{\mathbf{z}}$, were computed by Brey and Fertig [33]. In our geometry, the relevant surfaces are $\pm \hat{\mathbf{z}}$ and $\pm \hat{\mathbf{y}}$ where the projections of the spin operators follow Eqs. (2) and (4), yielding the effective Hamiltonians Eqs. (1) and (3), respectively. The parameters of surface Hamiltonians are then obtained from Eq. (20) [33] by projection. In particular, the band crossing energies of the $\hat{\mathbf{z}}$ and $\hat{\mathbf{y}}$ surfaces (which are the relevant surfaces for our choice of axes) are given by

$$
\begin{aligned}
& E_{0}(\hat{\mathbf{z}})=C+\xi M_{0}, \\
& E_{0}(\hat{\mathbf{y}})=C+\eta M_{0},
\end{aligned}
$$

and the corresponding Fermi velocities are given by

$$
\begin{gathered}
v_{F}(\hat{\mathbf{z}})=A_{2} \sqrt{1-\xi^{2}}, \\
v_{F, x}(\hat{\mathbf{y}})=A_{2} \sqrt{1-\eta^{2}}, \\
v_{F, z}(\hat{\mathbf{y}})=A_{1} \sqrt{1-\eta^{2}},
\end{gathered}
$$

where

$$
\xi=D_{1} / B_{1}, \quad \eta=D_{2} / B_{2} .
$$

In our numerical study, we use the tight-binding representation of the Hamiltonian in Eq. (20) and focus on a 3DTI wire attached to two semi-infinite leads [see Fig. 2(a)]. We evaluate nonequilibrium local spin densities $\left\langle S_{i}\right\rangle(m)=$ $\left\langle\psi_{\alpha}(m)\left|S_{i}\right| \psi_{\alpha}(m)\right\rangle$ for each site $m$, where $\psi_{\alpha}(m)$ is the wave function of the (occupied) state $\alpha$ at site $m$ and $S_{i}$ are the spin operators defined in Eq. (21). We then sum over all occupied states $\alpha$. For an infinitesimal bias, these are all scattering wave functions at a certain energy, $E_{F}$ originating from one of the leads, depending on the sign of the bias. Local charge density is similarly obtained when $S_{i} \rightarrow \sigma_{0} \tau_{0}$. We utilize the KWANT toolbox [50] for our numerical simulations. The parameters of our band Hamiltonian are chosen from the ab initio bandstructure calculations of $\mathrm{Bi}_{2} \mathrm{Se}_{3}$ [47] in our numerical simulations. The particular values used are $A_{1}=2.2 \mathrm{eV} \AA, A_{2}=$ $4.1 \mathrm{eV} \AA, B_{1}=10 \mathrm{eV}^{2}, B_{2}=56.6 \mathrm{eVA}^{2}, C=-0.0068 \mathrm{eV}$, $D_{1}=1.3 \mathrm{eV}^{2}, D_{2}=19.6 \mathrm{eV}^{2}$, and $M=0.28 \mathrm{eV}$. We have

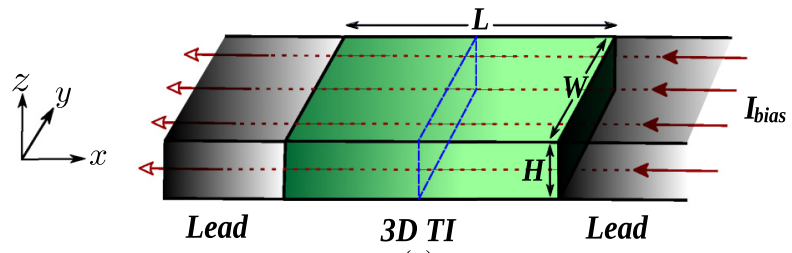

(a)

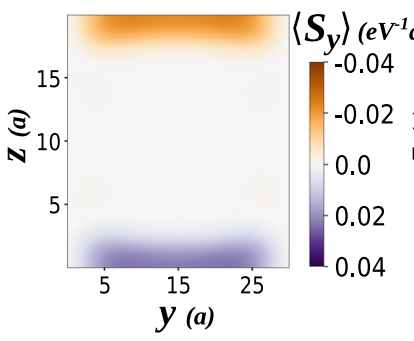

(b)

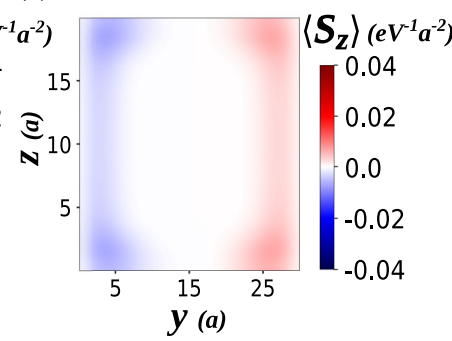

(c)
FIG. 2. Surface spin polarization of a 3DTI nanowire. (a) Sketch of a 3DTI nanowire attached to two semi-infinite leads. (b) $\left\langle S_{y}\right\rangle$ and (c) $\left\langle S_{z}\right\rangle$ denote the spatial profile of the averaged spin polarization (averaged over 1000 disorder configurations) along cross sections, oriented in the $\hat{\mathbf{x}}$ direction and marked as the blue rectangle in panel (a). Parameters used are $L=30 a, W=30 a, H=20 a, H_{\mathrm{SP}}=10 a$, $U_{0}=0.5 \mathrm{eV}$, and $E_{F}=0.15 \mathrm{eV}$, which is in the bulk gap.

also set the lattice constant to be $a=5 \AA$ in our numerical calculations.

\section{B. Spin dynamics and accumulation at the surface}

As a consequence of (pseudo)spin-momentum locking of the 3DTI surface states, the dynamics of spin and charge are coupled [51,52]. Thus, even nonmagnetic impurities can flip an electron's spin during scattering, leading to the dominant spin-relaxation mechanism. The situation here is similar to the so-called clean limit of the well-known spin-diffusion equations of a 2D electron gas with Rashba spin-orbit coupling [53], where the Dyakonov-Perel spin-relaxation time [54] is of the order of the momentum relaxation time. Hence, typical features of spin diffusion in standard semiconductors (which are in the "dirty" limit) such as the motional narrowing do not take place here. The steady-state spin-diffusion equation for the top (i.e., $\hat{\mathbf{z}}$ ) surface of a 3DTI is given by [51]

$$
\frac{\Sigma_{i}}{\tau}=\frac{D}{2} \nabla^{2} \Sigma_{i}+\left|\epsilon_{i j}\right| D \frac{\partial \Xi}{\partial x_{j}}-\epsilon_{i j} \frac{v_{F}}{2} \frac{\partial n}{\partial x_{j}},
$$

where $\Sigma_{i}, i=x, y$ are the in-plane components of the pseudospin density $\boldsymbol{\Sigma}, \boldsymbol{\Xi}=\partial \Sigma_{x} / \partial y+\partial \Sigma_{y} / \partial x$, and $n$ is the charge density. Furthermore, $\epsilon_{i j}$ is the $2 \mathrm{D}$ antisymmetric tensor and $D=v_{F}^{2} \tau / 2$ is the diffusion constant, with $\tau$ the momentum scattering time and $v_{F}$ the Fermi velocity $\left[v_{F}(\hat{\mathbf{z}})\right]$. We stress that these equations are valid only if the spin and charge accumulations vary appreciably at length scales much larger than the mean free path [52]. In the present paper, we only consider geometries where this condition is satisfied. In order to obtain the equations applicable to the side surfaces, $\left[v_{F}=v_{F, x}(\hat{\mathbf{y}})\right.$, see Appendix B], we generalize the diffusion equations to 


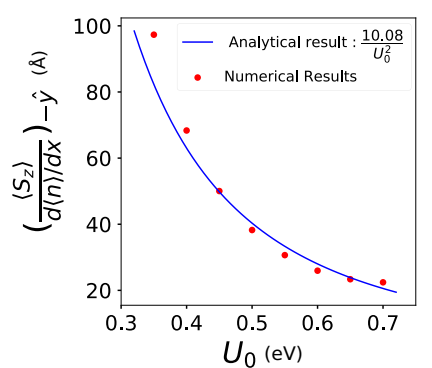

(a)

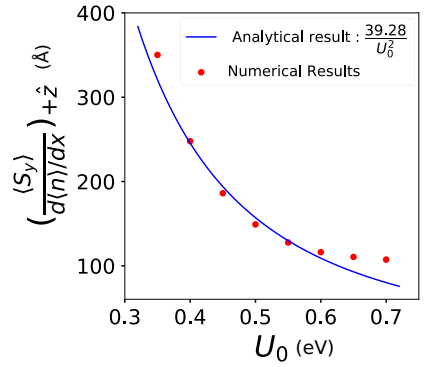

(b)
FIG. 3. Average ratios (a) $\left(\left\langle S_{z}\right\rangle / d\langle n\rangle / d x\right)_{-\hat{\mathbf{y}}} \quad$ and (b) $\left(\left\langle S_{y}\right\rangle / d\langle n\rangle / d x\right)_{+\hat{\mathbf{z}}}$ as a function of disorder strength $U_{0}$. The blue curves show the analytical and the red symbols the numerical results. Parameters in our simulations are $L=30 a$, $W=30 a, H=20 a$, and $E_{F}=0.15 \mathrm{eV}$, which is in the bulk gap.

anisotropic surfaces and obtain the dependence of the real spin density on the charge gradients due to the applied voltage bias:

$$
\begin{gathered}
\left(\frac{\left\langle S_{z}\right\rangle}{d\langle n\rangle / d x}\right)_{ \pm \hat{\mathbf{y}}}=\mp\left(\eta \frac{v_{F, x}(\hat{\mathbf{y}}) \tau}{2}\right)_{ \pm \hat{\mathbf{y}}}, \\
\left(\frac{\left\langle S_{y}\right\rangle}{d\langle n\rangle / d x}\right)_{ \pm \hat{\mathbf{z}}}= \pm\left(\frac{v_{F}(\hat{\mathbf{z}}) \tau}{2}\right)_{ \pm \hat{\mathbf{z}}}
\end{gathered}
$$

Hence, if $E_{F}$ sits in the bulk gap, then applying a bias voltage yields surface currents flowing in the $x$ direction, which in turn induces spin accumulations on the $\pm \hat{\mathbf{y}}$ and the $\pm \hat{\mathbf{z}}$ surfaces. This is the ISGE. In order to test these predictions, we numerically obtain spin densities via the method described in Sec. III A. Our results are shown in Figs. 2(b) and 2(c), where we plot the $x$-averaged cross-sectional profile for $\left\langle S_{y}\right\rangle$ and $\left\langle S_{z}\right\rangle$. Note that both components of the spin accumulation are localized to the respective surfaces and have opposite sign on opposite surfaces. Notice also that $\left\langle S_{x}\right\rangle=0$ in our configuration since it is along the current direction. Furthermore, $\left\langle S_{z}\right\rangle$ is smaller than $\left\langle S_{y}\right\rangle$ for $\eta<1$. The case $D_{2}=0$, as mentioned earlier, corresponds to a vanishing ISGE $\left\langle S_{z}\right\rangle$ and the "paradoxical" regime $\eta=0$ of Sec. II.

In order to test Eqs. (31) and (32) numerically, we consider the quotient on the left-hand side of these equations as a function of disorder strength $U_{0}$. Since in Fermi's "golden rule" regime $1 / \tau \sim U_{0}^{2}$, we expect a $U_{0}^{-2}$ behavior. In order to get the exact relation, we analytically calculate the mean free time using a $\mathbf{k} \cdot \mathbf{p}$ approximation for surface eigenmodes in Appendix B. Next, we perform numerical simulations and obtain the local spin or charge accumulations and average these over a square region in the middle of the $+\hat{\mathbf{z}}$ and $-\hat{\mathbf{y}}$ surfaces as well as over different disorder configurations with strength $U_{0}$. Finally, we compare our analytical prediction (the blue line) for the left-hand sides of Eqs. (31) and (32) against the numerical simulations (red dots) in Figs. 3(a) and 3(b), respectively. We find that our numerical results for ISGE are well described by the analytical formulas in Eqs. (31) and (32).

\section{Spin extraction}

Having discussed how spins can be induced at a topological insulator surface, we now study how these spins can

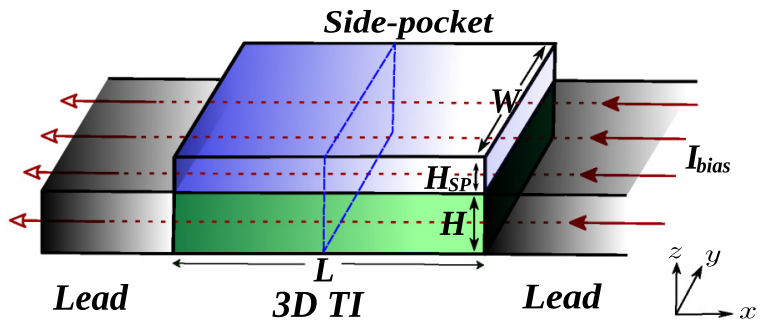

(a)

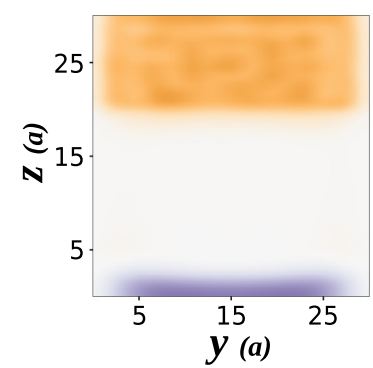

(b)

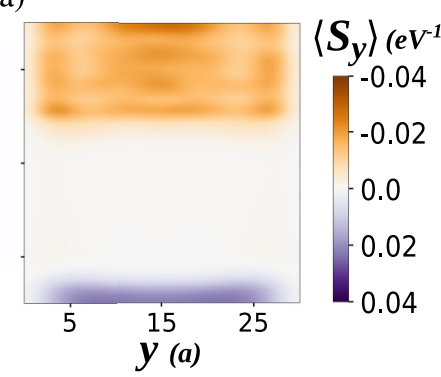

(c)
FIG. 4. Current-induced spin polarization into a side pocket at the top surface. $(\mathrm{a}-\mathrm{c})$ Spatial profile of the averaged spin polarization $\left\langle S_{y}(y, z)\right\rangle$ (averaged over 1000 disorder configurations) along cross sections in the $\hat{\mathbf{x}}$ direction shown as a dashed blue rectangle in panel (a). In panels (b) and (c) the side pockets are doped to hole bands $\left(V_{\text {gate }}=-0.8 \mathrm{eV}\right)$ and electron bands $\left(V_{\text {gate }}=0.9 \mathrm{eV}\right)$, respectively. Common parameters are $L=30 a, W=30 a, H=20 a, H_{\mathrm{SP}}=$ $10 a, U_{0}=0.5 \mathrm{eV}$, and $E_{F}=0.15 \mathrm{eV}$, which is in the bulk gap.

be extracted to be used in (presumably topologically trivial) spintronics circuitry. To this end, we focus on a geometry where a topologically trivial side pocket is attached to the TI nanowire [see Figs. 4(a) and 5(a)]. The current-induced spins at the TI surface can then leak into the side pocket, generating nonzero spin accumulation inside the side pocket. The nanowire size is chosen such that its length and width $L=$ $W=15 \mathrm{~nm}$ exceed the mean free path $l$, ensuring diffusive carrier dynamics. The mean free path is estimated in terms of the disorder potential strength $U_{0}$ using Fermi's "golden rule" (see Appendix B for details).

Spin extraction can take place at pockets that are attached to either surface of the 3DTI nanowire [see Figs. 4(a) and 5(a) for the geometry where the pocket is attached to the $\hat{\mathbf{z}}$ surface or the $\hat{\mathbf{y}}$ surface, respectively]. The pockets are gated in order to tune them to a metallic state, while charge carriers can be either electron- or holelike states; thus, there is a coupling only to the electron- or holelike spin-momentum locked components of the 3DTI surface states. The gating is modeled by adding a corresponding on-site energy term in the tight-binding grid while keeping the other parameters of the effective Hamiltonian unchanged.

We perform tight-binding simulations and numerically calculate the current-induced spin polarization $\left\langle S_{i}\right\rangle,(i=y, z)$, averaging over 1000 disorder configurations for a nanowire with side pockets. Figures 4(b) and 4(c) and Figs. 5(b) and 5 (c) show the spatial profile of the spin polarization along a perpendicular cross section for fixed doping values in the hole and electron bands, respectively. Focusing on the top ( $\hat{\mathbf{z}})$ surface, our simulations show all expected features: 


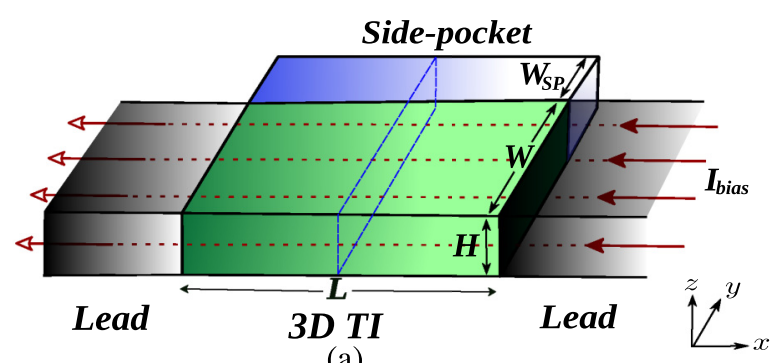

(a)

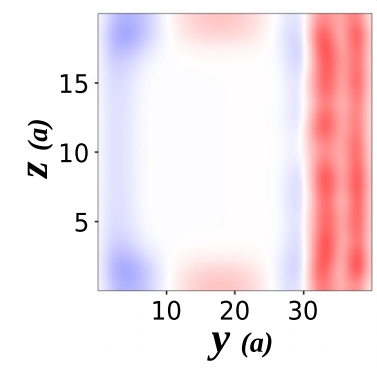

(b)

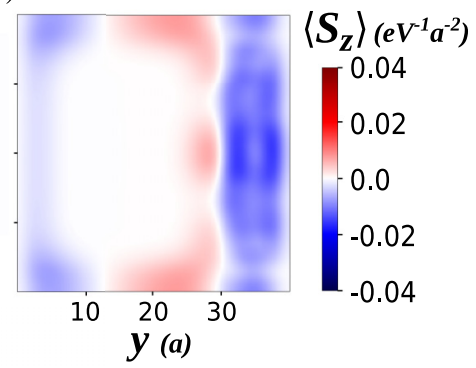

(c)
FIG. 5. Current-induced spin polarization into a side pocket at the side surface. (a) Sketch of a side pocket attached to the side surface of the system shown in Fig. 2(a). (b, c) Spatial profile of the averaged spin polarization $\left\langle S_{z}(y, z)\right\rangle$ (averaged over 1000 disorder configurations) along cross sections in the $\hat{\mathbf{x}}$ direction shown as a dashed blue rectangle in panel (a). In panels (b) and (c) the side pockets are doped to hole bands $\left(V_{\text {gate }}=-0.8 \mathrm{eV}\right)$ and electron bands $\left(V_{\text {gate }}=0.9 \mathrm{eV}\right)$, respectively. Common parameters are $L=30 a$, $W=30 a, H=20 a, W_{\mathrm{SP}}=10 a, U_{0}=0.5 \mathrm{eV}$, and $E_{F}=0.15 \mathrm{eV}$, which is in the bulk gap.

A substantial nonequilibrium spin accumulation can be extracted into the doped side pockets (Fig. 4). The extraction to the side ( $\hat{\mathbf{y}}$ ) surface (Fig. 5), on the other hand, has nontrivial features. We first note the somewhat surprising fact that even if the 3DTI surface has negligibly small spin accumulation, $\eta \approx 0$, the spin accumulation extracted into the side pocket is non-negligible (see corresponding figures in Appendix C). Furthermore, the extracted spin polarization changes sign when the gate voltage is tuned so that the charge carriers change from electrons to holes as can be seen from Figs. 5(b) and $5(\mathrm{c})$. We find that the geometry of the contact does not play a crucial role as it does for a $2 \mathrm{D}$ electron gas with Rashba spin-orbit coupling: In that case, wide contacts lead to reduced extraction [28] while for TIs wider contacts lead to enhanced extraction. In order to further study the spin-gate effect mentioned above, we plot the spin accumulation $\left\langle S_{z}\right\rangle$ averaged over the side pocket, as a function of the gate voltage applied to the side pocket, in Fig. 6. We find that the spin accumulation depends linearly on the gate voltage and the sign of polarization changes by switching the side pocket polarity from hole to electron type.

Finally, we show that one can locally control the polarization direction of different parts of side pockets by local gating. In Fig. 7, we apply the local gate profile where the electron puddles change into hole puddles within a region much smaller than the spin precession length $\ell_{\mathrm{SP}}$. We find that the spatial profile of the polarization of the extracted spin accumulation closely follows the local gate potential. Thus,

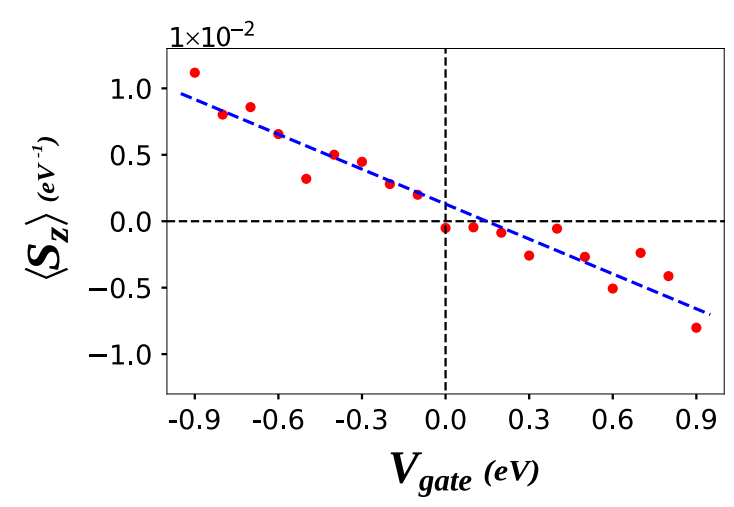

FIG. 6. Gate dependence of side pocket spin polarization. $\left\langle S_{z}\right\rangle$ averaged over 1000 disordered configurations and doped side pocket sites is plotted vs gate potential. We consider the following parameters: $L=30 a, W=30 a, H=20 a W_{\mathrm{SP}}=10 a, U_{0}=0.5 \mathrm{eV}$, and $E_{F}=0.15 \mathrm{eV}$, which is in the bulk gap. The blue line is the best-fitted line.

we show that it is possible to electrically control local spin polarization within length scales much smaller than the spin precession length.

\section{CONCLUSIONS}

In conclusion, we focus on the current-induced spins at the surfaces of 3DTIs and show how to extract these spins into topologically trivial materials commonly used in electronic devices. We find that unlike the corresponding effect in 2D electron gases with Rashba spin-orbit interaction the mixing of the electron and hole degrees of freedom at the TI surface allows for additional methods for spin manipulation. In particular, we exposed a way to use electrical gate potentials to locally manipulate spins in regions smaller than the spin precession length. The extracted spins can then be detected via usual spintronics methods such as attaching a ferromagnetic lead or using magneto-optical Kerr microscopy. This opens up new possibilities for spin manipulation in spintronics devices.

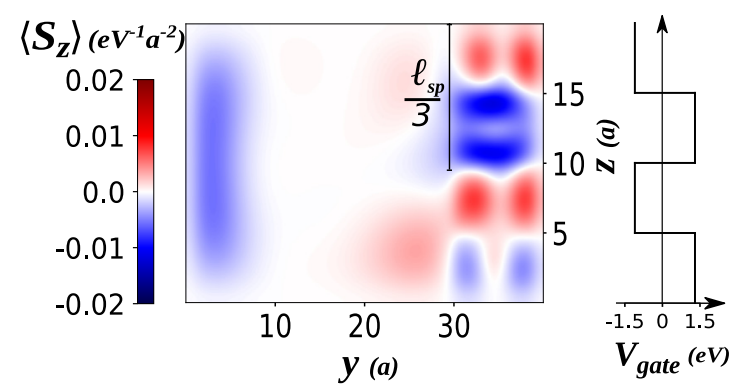

FIG. 7. Spatial profile of the averaged spin polarization $\left\langle S_{z}(y, z)\right\rangle$ (averaged over 1000 disorder configurations) along cross sections in the $\hat{\mathbf{x}}$ direction for the system shown in Fig. 5(a). The side pocket is alternatively doped to electron bands $\left(V_{\text {gate }}=1.3 \mathrm{eV}\right.$ ) and hole bands $\left(V_{\text {gate }}=-1.1 \mathrm{eV}\right)$. The side pocket is divided into four parts in the $\hat{\mathbf{z}}$ direction and the spatial profile is averaged over $\hat{\mathbf{x}}$ planes. Spin precession length in the $\hat{\mathbf{z}}$ direction, $\ell_{\mathrm{SP}} \simeq 32 a$ (considering $B_{1}=$ $B_{2}$ ). Other parameters used are $W_{\mathrm{SP}}=10 a, L=30 a, W=30 a$, $H=20 a, U_{0}=0.7 \mathrm{eV}$, and $E_{F}=0.15 \mathrm{eV}$, which is in the bulk gap. 


\section{ACKNOWLEDGMENTS}

A.A. thanks B. Pekerten and V. Sazgari for helpful conversations. This work was supported by the Scientific and Technological Research Council of Turkey under Grant No. 114F163 and by the Deutsche Forschungsgemeinschaft under Project No. 314695032 SFB 1277 (Subproject No. A07).

\section{APPENDIX A: EFFECTIVE SURFACE HAMILTONIANS AND SPIN OPERATORS}

Surface states in 3DTIs decay exponentially into the bulk and have energies in the bulk band gap. We first consider a semi-infinite 3DTI system situated in $z \geqslant 0(z \leqslant 0)$ with a surface normal $-\hat{\mathbf{z}}$ ( $\hat{\mathbf{z}})$ pointing away from the bulk. By considering a vanishing boundary condition at the surface, eigenfunctions corresponding to these states can be written as

$$
\phi \sim u\left(k_{x}, k_{y}, \lambda_{1,2}\right) e^{i\left(k_{x} x+k_{y} y\right)}\left(e^{ \pm \lambda_{1} z}-e^{ \pm \lambda_{2} z}\right),
$$

where the \pm sign in the $z$ direction corresponds to a system with a surface normal in the $\mp \hat{\mathbf{z}}$ direction at $z=0$. Here $\operatorname{Re}\left(\lambda_{1,2}\right)>0$ and $u\left(k_{x}, k_{y}, \lambda_{1,2}\right)$ is a spinor that is an eigenstate of the 3DTI Hamiltonian described in Eq. (20), corresponding to $k_{z}=-i \lambda_{1,2}$ :

$$
u^{ \pm \hat{\mathbf{z}}}=\frac{1}{\sqrt{2}}\left[\begin{array}{c}
\sqrt{1+\xi} \\
\mp i \sqrt{1-\xi} \\
0 \\
0
\end{array}\right], \quad v^{ \pm \hat{\mathbf{z}}}=\frac{1}{\sqrt{2}}\left[\begin{array}{c}
0 \\
0 \\
\sqrt{1+\xi} \\
\pm i \sqrt{1-\xi}
\end{array}\right]
$$

with energy dispersion to the lowest order of $k$ given by

$$
E^{ \pm}=C+\xi M_{0} \pm A_{2} \sqrt{1-\xi^{2}} k_{\perp}
$$

where $k_{\perp}^{2}=k_{x}^{2}+k_{y}^{2}$. Hence, the effective surface Hamiltonian as given in the text is obtained through projecting the 3DTI Hamiltonian in basis states given in Eq. (A1) and using the spinor eigenstates stated in Eq. (A2). To lowest order in $k_{x}$ and $k_{y}$, this results in

$$
H^{ \pm \hat{\mathbf{z}}}=C+\xi M_{0} \pm A_{2} \sqrt{1-\xi^{2}}\left(\begin{array}{cc}
0 & i k_{x}+k_{y} \\
-i k_{x}+k_{y} & 0
\end{array}\right),
$$

which is introduced as Eq. (1) in the paper. The real spin operators for the $\hat{z}$ surface are formed by projecting the spin operators in the basis of bulk states, Eq. (21), onto the two surface states

$$
s_{x}=\sigma_{x}, \quad s_{y}=\sigma_{y}, \quad s_{z}=\sigma_{z},
$$

which is stated as Eq. (2). The effective surface Hamiltonians and real spin operators corresponding to other surfaces can be calculated similarly.

\section{APPENDIX B: MEAN FREE TIME ESTIMATION}

We proceed with a Fermi's "golden rule" estimation of the mean free path. The surface modes are four-spinors with $k$ dependent components [55] due to (pseudo)spin-momentum coupling. Such $k$ dependence can lead to substantial differences between lifetime and transport time [56]. In the case of uncorrelated disorder, however, the difference is only an $O(1)$ factor [52] and thus irrelevant for our estimations. We

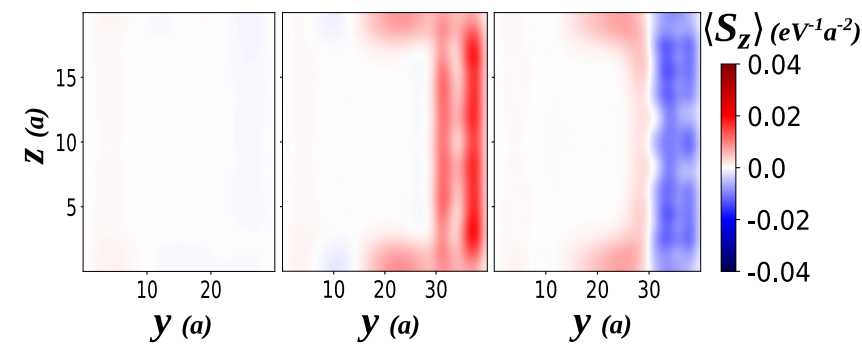

(a)

(b)

(c)

FIG. 8. Current-induced spin polarization into a side pocket at the side surface when $\eta=0$. Spatial profile of the averaged spin polarization $\left\langle S_{z}(y, z)\right\rangle$ (averaged over 1000 disorder configurations) along cross sections in the $\hat{\mathbf{x}}$ direction. (a) $\left\langle S_{z}(y, z)\right\rangle$ corresponds to the system shown in Fig. 2(a). (b, c) $\left\langle S_{z}(y, z)\right\rangle$ corresponds to the system shown in Fig. 5(a). In panels (b) and (c) the side pockets, $W_{\mathrm{SP}}=10 a$, are doped to hole bands $\left(V_{\text {gate }}=-0.7 \mathrm{eV}\right)$ and electron bands $\left(V_{\text {gate }}=0.7 \mathrm{eV}\right)$, respectively. Common parameters are $L=$ $30 a, W=30 a, H=20 a, U_{0}=0.5 \mathrm{eV}$, and $E_{F}=0.15 \mathrm{eV}$, which is in the bulk gap. We set $D_{2}=0$ in all parts of the system.

thus work exclusively with band-bottom $k=0$ spinors. We consider a TI slab extended in $x$ and $y$ directions, having a length $L$ and a width $W$ along the $x$ direction and $y$ direction, respectively, and a thickness $H$ along the $z$ direction. We further assume white-noise disorder of the form $\left\langle V(\boldsymbol{r}) V\left(\boldsymbol{r}^{\prime}\right)\right\rangle=$ $\gamma \delta\left(\boldsymbol{r}-\boldsymbol{r}^{\prime}\right)$. Therefore, using spinors stated in Eq. (A2) leads to

$$
\left\langle\left|V_{k k^{\prime}}\right|^{2}\right\rangle=\frac{\gamma}{L W} \frac{\alpha}{\beta^{2}}
$$

where $\alpha=\int_{0}^{H} d z f^{2}(z), \beta=\int_{0}^{H} d z f(z)$ with $f(z)=\left(e^{-\lambda_{1}^{*} z}-\right.$ $\left.e^{-\lambda_{2}^{*} z}\right)\left(e^{-\lambda_{1} z}-e^{-\lambda_{2} z}\right)$. We use Fermi's "golden rule" to derive the inverse mean free time and find

$$
\frac{1}{\tau}=\sum_{k^{\prime}} \frac{1}{\tau\left(k \rightarrow k^{\prime}\right)}=\frac{2 \pi}{\hbar} \sum_{k^{\prime}}\left\langle\left|V_{k k^{\prime}}\right|^{2}\right\rangle \delta\left(E_{k}-E_{k^{\prime}}\right)
$$

for surface states of a disordered 3DTI with semi-infinite boundary condition in the $\hat{z}$ direction, i.e., $H \longrightarrow \infty$. Based on Eq. (A3), we have

$$
\delta\left(E_{k}^{+}-E_{k^{\prime}}^{+}\right)=\frac{1}{A_{2} \sqrt{1-\xi^{2}}} \delta\left(k_{\perp}^{\prime}-\frac{E^{+}-C-\xi M_{0}}{A_{2} \sqrt{1-\xi^{2}}}\right) .
$$

Hence, the resulting total ensemble-averaged mean free time of surface states on the $\hat{z}$ surface reads

$$
\frac{1}{\tau}=\frac{2 \gamma}{\hbar} \frac{E^{+}-C-\xi M_{0}}{A_{2}^{2}\left(1-\xi^{2}\right)} \frac{\alpha}{\beta^{2}} .
$$

Here, since $\lambda$ 's are complex conjugate partners in our system, $\lambda_{1}=\lambda_{2}^{*}=\tilde{a}+i \tilde{b}$, we find $\alpha=\frac{3 \tilde{b}^{4}}{8 \tilde{a}^{5}+10 \tilde{a}^{3} \tilde{b}^{2}+2 \tilde{a} \tilde{b}^{4}}$ and $\beta=$ $\frac{\tilde{b}^{2}}{\tilde{a}^{3}+\tilde{a} \tilde{b}^{2}}$.

Similarly, for an energy dispersion, to the lowest order of $k$, for $\hat{y}$-plane surface states,

$$
E^{ \pm}=C+\eta M_{0} \pm \sqrt{1-\eta^{2}} \sqrt{A_{2}^{2} k_{x}^{2}+A_{1}^{2} k_{z}^{2}},
$$


we obtain the total ensemble-averaged inverse mean free time

$$
\frac{1}{\tau} \simeq \frac{2 \gamma}{\hbar} \frac{E^{+}-C-\eta M_{0}}{A_{2}^{2}\left(1-\eta^{2}\right)} \frac{\alpha^{\prime}}{\beta^{\prime 2}}
$$

where we approximate the Fermi velocity, $v_{F}=v_{F, x}(\hat{\mathbf{y}})$, at this surface based on Eq. (B5) since $A_{2}>A_{1}$. Note that $\alpha(\beta)$ and $\alpha^{\prime}\left(\beta^{\prime}\right)$ are different values since the depths of the surface states into the bulk in different surfaces are not the same according to the parameters of the Hamiltonian.

According to our mean free time and Fermi velocities derivations, Eqs. (31) and (32) yield

$$
\left(\frac{\left\langle S_{z}\right\rangle}{d\langle n\rangle / d x}\right)_{-\hat{\mathbf{y}}}=\left(\frac{\eta\left(A_{2} \sqrt{1-\eta^{2}}\right)^{3}}{E^{+}-C-\eta M_{0}} \frac{\beta^{\prime 2}}{4 \alpha^{\prime} \gamma}\right)_{-\hat{\mathbf{y}}},
$$

$$
\left(\frac{\left\langle S_{y}\right\rangle}{d\langle n\rangle / d x}\right)_{+\hat{\mathbf{z}}}=\left(\frac{\left(A_{2} \sqrt{1-\xi^{2}}\right)^{3}}{E^{+}-C-\xi M_{0}} \frac{\beta^{2}}{4 \alpha \gamma}\right)_{+\hat{\mathbf{z}}},
$$

where $\gamma=U_{0}^{2} a^{3}$ in the discretized system.

\section{APPENDIX C: $\eta=0$ CASE}

Here we provide figures for the case $D_{2}=0$ leading to $\eta=0$. It is clearly seen that while there is negligible spin accumulation on the side of a 3DTI [Fig. 8(a)] spin extraction is non-negligible in the side pocket and spin polarization can be switched via a gate potential [see Figs. 8(b) and 8(c)].
[1] I. Žutić, J. Fabian, and S. D. Sarma, Rev. Mod. Phys. 76, 323 (2004).

[2] D. C. Ralph and M. D. Stiles, J. Magn. Magn. Mater. 320, 1190 (2008).

[3] S. A. Wolf, D. D. Awschalom, R. A. Buhrman, J. M. Daughton, S. von Molnar, M. L. Roukes, A. Y. Chtchelkanova, and D. M. Treger, Science 294, 1488 (2001).

[4] K. Sato and H. Katayama-Yoshida, Semicond. Sci. Technol. 17, 367 (2002).

[5] S. Datta and B. Das, Appl. Phys. Lett. 56, 665 (1990).

[6] D. D. Awschalom, D. Loss, and N. Samarth, Semiconductor Spintronics and Quantum Computation (Springer, New York, 2013).

[7] Y. K. Kato, R. C. Myers, A. C. Gossard, and D. D. Awschalom, Science 306, 1910 (2004).

[8] J. Schliemann, Rev. Mod. Phys. 89, 011001 (2017).

[9] V. M. Edelstein, Solid State Commun. 73, 233 (1990).

[10] J. I. Inoue, G. E. W. Bauer, and L. W. Molenkamp, Phys. Rev. B 67, 033104 (2003).

[11] A. Y. Silov, P. A. Blajnov, J. H. Wolter, R. Hey, K. H. Ploog, and N. S. Averkiev, Appl. Phys. Lett. 85, 5929 (2004).

[12] S. D. Ganichev, S. N. Danilov, P. Schneider, V. V. Bel'Kov, L. E. Golub, W. Wegscheider, D. Weiss, and W. Prettl, J. Magn. Magn. Mater. 300, 127 (2006).

[13] M. I. D'yakonov and V. I. Perel, Pis'ma Zh. Eksp. Teor. Fiz. 13, 657 (1971) [JETP Lett. 13, 467 (1971)].

[14] M. I. D'yakonov and V. I. Perel, Phys. Lett. A 35, 459 (1971).

[15] J. E. Hirsch, Phys. Rev. Lett. 83, 1834 (1999).

[16] M. Governale, F. Taddei, and R. Fazio, Phys. Rev. B 68, 155324 (2003)

[17] A. G. Mal'shukov, C. S. Tang, C. S. Chu, and K. A. Chao, Phys. Rev. B 68, 233307 (2003).

[18] S. Murakami, N. Nagaosa, and S. C. Zhang, Phys. Rev. B 69, 235206 (2004).

[19] M. Scheid, M. Kohda, Y. Kunihashi, K. Richter, and J. Nitta, Phys. Rev. Lett. 101, 266401 (2008).

[20] A. G. Aronov, Y. B. Lyanda-Geller, and G. E. Pikus, Zh. Eksp. Teor. Fiz. 100, 973 (1991) [Sov. Phys. JETP 73, 537 (1991)].

[21] E. Saitoh, M. Ueda, H. Miyajima, and G. Tatara, Appl. Phys. Lett. 88, 182509 (2006).

[22] T. Kimura, Y. Otani, T. Sato, S. Takahashi, and S. Maekawa, Phys. Rev. Lett. 98, 156601 (2007).
[23] K. Uchida, S. Takahashi, K. Harii, J. Ieda, W. Koshibae, K. Ando, S. Maekawa, and E. Saitoh, Nature (London) 455, 778 (2008).

[24] S. O. Valenzuela and M. Tinkham, Nature (London) 442, 176 (2006).

[25] T. Seki, Y. Hasegawa, S. Mitani, S. Takahashi, H. Imamura, S. Maekawa, J. Nitta, and K. Takanashi, Nat. Mater. 7, 125 (2008).

[26] S. D. Ganichev, E. L. Ivchenko, V. V. Bel'kov, S. A. Tarasenko, M. Sollinger, D. Weiss, W. Wegscheider, and W. Prettl, Nature (London) 417, 153 (2002).

[27] J. C. Rojas Sánchez, L. Vila, G. Desfonds, S. Gambarelli, J. P. Attané, J. M. De Teresa, C. Magén, and A. Fert, Nat. Commun. 4, 2944 (2013).

[28] İ. Adagideli, M. Scheid, M. Wimmer, G. E. W. Bauer, and K. Richter, New J. Phys. 9, 382 (2007).

[29] I. Žutić, J. Fabian, and S. Das Sarma, Phys. Rev. Lett. 88, 066603 (2002).

[30] K. Shen, G. Vignale, and R. Raimondi, Phys. Rev. Lett. 112, 096601 (2014).

[31] F. Zhang, C. L. Kane, and E. J. Mele, Phys. Rev. B 86, 081303(R) (2012).

[32] P. G. Silvestrov, P. W. Brouwer, and E. G. Mishchenko, Phys. Rev. B 86, 075302 (2012).

[33] L. Brey and H. A. Fertig, Phys. Rev. B 89, 085305 (2014).

[34] H. Zhang, C. Liu, X. Qi, X. Dai, Z. Fang, and S. Zhang, Nat. Phys. 5, 438 (2009).

[35] Y. L. Chen, J. G. Analytis, J.-H. Chu, Z. K. Liu, S.-K. Mo, X. L. Qi, H. J. Zhang, D. H. Lu, X. Dai, Z. Fang, S. C. Zhang, I. R. Fisher, Z. Hussain, and Z.-X. Shen, Science 325, 178 (2009).

[36] Y. Xia, D. Qian, D. Hsieh, L. Wray, A. Pal, H. Lin, A. Bansil, D. Grauer, Y. S. Hor, R. J. Cava, and M. Z. Hasan, Nat. Phys. 5, 398 (2009).

[37] Z. Alpichshev, J. G. Analytis, J.-H. Chu, I. R. Fisher, Y. L. Chen, Z. X. Shen, A. Fang, and A. Kapitulnik, Phys. Rev. Lett. 104, 016401 (2010).

[38] T. Zhang, P. Cheng, X. Chen, J.-F. Jia, X. Ma, K. He, L. Wang, H. Zhang, X. Dai, Z. Fang, X. Xie, and Q.-K. Xue, Phys. Rev. Lett. 103, 266803 (2009).

[39] P. Cheng, C. Song, T. Zhang, Y. Zhang, Y. Wang, J.-F. Jia, J. Wang, Y. Wang, B.-F. Zhu, X. Chen, X. Ma, K. He, L. Wang, X. Dai, Z. Fang, X. Xie, X.-L. Qi, C.-X. Liu, S.-C. Zhang, and Q.-K. Xue, Phys. Rev. Lett. 105, 076801 (2010).

[40] T. Hanaguri, K. Igarashi, M. Kawamura, H. Takagi, and T. Sasagawa, Phys. Rev. B 82, 081305(R) (2010). 
[41] Z. Alpichshev, J. G. Analytis, J.-H. Chu, I. R. Fisher, and A. Kapitulnik, Phys. Rev. B 84, 041104(R) (2011).

[42] D. Hsieh, Y. Xia, L. Wray, D. Qian, A. Pal, J. H. Dil, J. Osterwalder, F. Meier, G. Bihlmayer, C. L. Kane, Y. S. Hor, R. J. Cava, and M. Z. Hasan, Science 323, 919 (2009).

[43] E. L. Ivchenko, Y. B. Lyanda-Geller, and G. E. Pikus, Zh. Eksp. Teor. Fiz. 98, 989 (1990) [Sov. Phys. JETP 71, 550 (1990)].

[44] Spintronics Handbook, Second Edition: Spin Transport and Magnetism, edited by E. Y. Tsymbal and I. Žutic (CRC Press, Boca Raton, 2019).

[45] C. Gorini, A. Maleki Sheikhabadi, K. Shen, I. V. Tokatly, G. Vignale, and R. Raimondi, Phys. Rev. B 95, 205424 (2017).

[46] Y. Ando and M. Shiraishi, J. Phys. Soc. Jpn. 86, 011001 (2017).

[47] C.-X. Liu, X.-L. Qi, H. J. Zhang, X. Dai, Z. Fang, and S.-C. Zhang, Phys. Rev. B 82, 045122 (2010).

[48] J. H. Bardarson, P. W. Brouwer, and J. E. Moore, Phys. Rev. Lett. 105, 156803 (2010).
[49] J. Ziegler, R. Kozlovsky, C. Gorini, M.-H. Liu, S. Weishäupl, H. Maier, R. Fischer, D. A. Kozlov, Z. D. Kvon, N. Mikhailov, S. A. Dvoretsky, K. Richter, and D. Weiss, Phys. Rev. B 97, 035157 (2018).

[50] C. W. Groth, M. Wimmer, A. R. Akhmerov, and X. Waintal, New J. Phys. 16, 063065 (2014).

[51] A. A. Burkov and D. G. Hawthorn, Phys. Rev. Lett. 105, 066802 (2010).

[52] P. Schwab, R. Raimondi, and C. Gorini, Europhys. Lett. 93, 67004 (2011).

[53] E. G. Mishchenko, A. V. Shytov, and B. I. Halperin, Phys. Rev. Lett. 93, 226602 (2004).

[54] M. I. D’yakonov and V. I. Perel, Fiz. Tverd. Tela 13, 3581 (1971) [Sov. Phys. Solid State 13, 3023 (1972)].

[55] W.-Y. Shan, H.-Z. Lu, and S.-Q. Shen, New J. Phys. 12, 043048 (2010).

[56] D. Culcer, E. H. Hwang, T. D. Stanescu, and S. Das Sarma, Phys. Rev. B 82, 155457 (2010). 